\title{
Polinização do dendezeiro por besouros no Sul da Bahia
}

\author{
José Inácio Lacerda Moura( ${ }^{(1)}$, Francisco Jorge Cividanes ${ }^{(2)}$, Lindolfo Pereira dos Santos Filho ${ }^{(1)}$ \\ e Raul Rene Valle ${ }^{(1)}$
}

(1)Comissão Executiva do Plano da Lavoura Cacaueira, Caixa postal 07, CEP 45690-970 Itabuna, BA. E-mail: jinaciolacerda@yahoo.com.br, lindolfo@cepec.gov.br, raul@cepec.gov.br (2)Universidade Estadual Paulista, Departamento de Fitossanidade, Via de acesso Prof. Paulo Donato Castellane, s/no, CEP 14884-900 Jaboticabal, SP. E-mail: fjcivida@fcav.unesp.br

\begin{abstract}
Resumo - O objetivo deste trabalho foi determinar a taxa de fecundação de dendezeiros, após introdução de Elaeidobius kamerunicus, no Sul da Bahia, e a flutuação populacional, distribuição espacial e a influência de fatores meteorológicos sobre E. kamerunicus e E. subvittatus. Análises físicas de 12 mil espiguetas de dendezeiros foram realizadas em 2004-2006. Na avaliação da relação entre a temperatura média mensal e o total mensal de indivíduos de E. kamerunicus e E. subvittatus, utilizou-se a correlação de Pearson. A taxa de fecundação foi de $79,4 \%$, um incremento de $19 \%$ quando comparado aos dendezeiros polinizados apenas por $E$. subvittatus. Plantios comerciais de dendezeiros em Ituberá e Nazaré, BA, apresentaram taxas médias de fecundações de $76 \%$. E. kamerunicus foi dominante nos plantios de dendezeiro de Una, BA. Populações de E. kamerunicus sobrepujaram as de E. subvittatus, em todos os municípios onde existem grandes maciços de dendezeiros subespontâneos. No entanto, em Itapebi, BA, as populações de E. subvittatus superaram as de E. kamerunicus. A temperatura e a precipitação pluvial afetam a atividade de vôo e densidade populacional de E. subvittatus.
\end{abstract}

Termos para indexação: Elaeidobius kamerunicus, Elaeidobius subvittatus, Elaeis guineensis, condições meteorológicas, flutuação populacional, taxa de fecundação.

\section{Pollination of oil palm by weevils in Southern Bahia, Brazil}

\begin{abstract}
The objective of this work was to determine the fertilization rate of oil palm trees, after the introduction of Elaeidobius kamerunicus in Southern Bahia, Brazil, and the population fluctuation, spatial distribution and the influence of meteorological factors on E. kamerunicus and E. subvittatus. Physical analyses of 12 thousand oil palm spikelets were accomplished during 2004-2006. Pearson correlation was used to evaluate the relation between monthly average temperatures and monthly totals of E. kamerunicus and E. subvittatus individuals. The fertilization rate was $79.4 \%$, an increase of $19 \%$ when compared to palm trees pollinated only by $E$. subvittatus. Commercial plantations of oil palm, in Ituberá and Nazaré, BA, presented $76 \%$ mean rates of fertilization. E. kamerunicus was the dominant species in the plantations of Una, BA. Populations of E. kamerunicus overwhelmed $E$. subvittatus ones in all the municipalities where there are great areas of subspontaneous palm oil. However, in Itapebi, BA, populations of $E$. subvittatus exceeded those of $E$. kamerunicus. Temperature and rainfall affected the flight activity and the population density of $E$. subvittatus.
\end{abstract}

Index terms: Elaeidobius kamerunicus, Elaeidobius subvittatus, Elaeis guineensis, meteorological factors, population fluctuation, fecundation rate.

\section{Introdução}

O dendezeiro, Elaeis guineensis Jacquin, é uma planta monóica, ou seja, as flores masculinas e femininas são produzidas na mesma planta, mas separadas, em inflorescências masculinas e femininas. Essa característica favorece a polinização cruzada, pois raramente as flores femininas e masculinas ficam simultaneamente receptivas na mesma planta (Conceição \& Muller, 2000).
$\mathrm{Na}$ América Latina, os insetos polinizadores mais importantes associados ao dendezeiro pertencem à família Nitidulidae (Mystrops costaricensis Gillogly) e Curculionidae (Elaeidobius subvittatus Faust). É possível que E. subvittatus tenha sido introduzido no Brasil no século XVI, por ocasião do tráfego marítimo entre a África e o Brasil (Genty et al., 1986). Essa espécie distingue-se como polinizador mais eficiente que M. costaricensis, por apresentar maior capacidade de 
transportar pólen e ser mais ativo nos períodos de intensa luminosidade (Genty et al., 1986).

Na Malásia, onde o dendê é exótico, a polinização assistida era requerida até 1981, ano em que Elaeidobius kamerunicus Faust foi introduzido, proveniente de Camarões, África, o que permitiu a suspensão da polinização assistida e aumentou a produção do óleo, pelo aumento na fecundação dos cachos (Syed et al., 1982). Assim, após a introdução de E. kamerunicus na Malásia, a taxa de fecundação do dendezeiro aumentou de 20 para $50-70 \%$ (Harun \& Noor, 2002).

Entre 1984 e 1986, E. kamerunicus foi introduzido na Colômbia, Equador, Costa Rica e Honduras, com resultado altamente favorável, ou seja, houve aumento na taxa de fecundação dos dendezeiros (Chinchilla et al., 1990).

Prada et al. (1998) estudaram a efetividade das espécies de Elaeidobius na polinização do dendezeiro na Venezuela. De acordo com os autores, 31.321 indivíduos de E. kamerunicus e 578 indivíduos de $E$. subvittatus visitaram uma inflorescência feminina, durante um dia de antese, e a média de 446 e 985 grãos de pólen foram transportados por fêmeas e machos de E. kamerunicus, respectivamente, enquanto machos de E. subvittatus transportaram 246 e fêmeas 116 grãos de pólen.

Levando-se em consideração os resultados obtidos em outros países (Chinchilla et al., 1990), em 1994 a Comissão Executiva do Plano da Lavoura Cacaueira (Ceplac) e a Embrapa Amazônia Oriental introduziram em Una, BA, espécimes de E. kamerunicus oriundos de Belém, PA, para melhorar a taxa de fecundação dos dendezeiros da região.

O objetivo deste trabalho foi determinar a taxa de fecundação de dendezeiros, após introdução de E. kamerunicus no Sul da Bahia, e a flutuação populacional, distribuição espacial e a influência de fatores meteorológicos sobre E. kamerunicus e E. subvittatus.

\section{Material e Métodos}

O trabalho foi desenvolvido na Estação Experimental Lemos Maia (Esmai), da Ceplac, no Município de Una, BA $\left(15^{\circ} 17^{\prime} \mathrm{S}, 39^{\circ} 4^{\prime} \mathrm{W}\right)$. O clima da região caracteriza-se como quente e úmido, sem estação seca definida (tipo Af), conforme Köppen.

E. kamerunicus foi introduzido em Una, BA, em 1994, por meio de espiguetas da inflorescência masculina, de dendezeiros provenientes da Embrapa Amazônia Oriental, Belém, PA, com larvas desse inseto em seu interior. As espiguetas foram tratadas com solução de Helmibem a $0,1 \%$ e, posteriormente, colocadas em gaiolas para a obtenção de formas adultas. Após seis meses de quarentena e obtenção de aproximadamente seis gerações de E. kamerunicus, em laboratório, 8.540 indivíduos foram liberados no banco de germoplasma de dendê da Esmai. O banco de germoplasma apresenta área de 16 ha, e é constituído por diversas progênies de dendezeiros, entre as quais Dura, Psifera, Tenera, E. oleifera e híbridos interespecíficos.

As análises dos cachos de dendezeiros tiveram início após dez anos da liberação de E. kamerunicus. Os cachos foram colhidos em uma área experimental de um hectare da variedade Tenera, com idade de nove anos e instalada próxima ao banco de germoplasma.

A percentagem de frutos normais por cacho, ou taxa de fecundação, foi determinada pela análise de 600 cachos de dendezeiro, utilizando a fórmula: $\%$ frutos normais $=100$ (total de frutos normais/total de frutos) (Chinchilla et al., 1990). O número total de frutos foi obtido pela soma do número de frutos normais (com amêndoa) e de frutos partenocárpicos.

Os cachos colhidos foram levados para o laboratório. Depois de pesados, todas as espiguetas foram retiradas do pedúnculo e misturadas; em seguida, foram retiradas amostras de vinte espiguetas, de forma aleatória, num total de 12.000 espiguetas amostradas. Com auxílio de uma faca, os frutos foram abertos, a fim de identificar aqueles com amêndoa e os partenocárpicos. As flores abortivas foram consideradas partenocárpicas.

A avaliação da densidade populacional de E. kamerunicus e E. subvittatus, sobre as inflorescências masculinas de dendezeiros, foi efetuada uma vez por mês, entre $8 \mathrm{~h}$ e $12 \mathrm{~h}$. Para isso, colheramse cinco inflorescências masculinas em estádio de antese, que foram fortemente agitadas dentro de saco de plástico, para a separação dos insetos polinizadores. Com um cilindro de $43 \mathrm{~cm}^{3}$, foi retirada uma amostra de pólen bruto (estames + pólen + insetos), que foi secada em estufa para posterior contagem dos insetos. 
Registros de precipitação pluvial, temperatura e umidade relativa do ar foram obtidos na estação meteorológica da Esmai. Utilizou-se a correlação de Pearson, na avaliação da relação entre a temperatura média mensal e os totais mensais de E. kamerunicus e E. subvittatus.

$\mathrm{Na}$ determinação da distribuição espacial de E. kamerunicus, e na quantificação de sua população em relação a E. subvittatus, foram realizados levantamentos populacionais dessas espécies em plantios de dendezeiros comerciais e subespontâneos, a 250 km ao norte do local de soltura dos espécimes de E. kamerunicus em 1994, nos municípios de Ituberá, Taperoá, Valença e Nazaré, BA, em setembro de 2006. No sentido sul, a cerca de $350 \mathrm{~km}$ do local de soltura de E. kamerunicus, foram realizados levantamentos populacionais em área experimental de 50 ha de dendezeiros, no município de Itapebi, BA. A coleta dos insetos foi realizada no período da manhã. As inflorescências masculinas, no estádio de antese, foram colhidas, para se determinar o número de espécimes de E. kamerunicus e E. subvittatus presentes, segundo método de estudo da densidade populacional de E. kamerunicus e E. subvittatus descrito anteriormente.

Concomitantemente aos levantamentos, foi avaliada a taxa de fecundação dos dendezeiros em plantios comerciais. Entre agosto e setembro de 2006, foram colhidos de forma aleatória 20 cachos de dendezeiros, nos plantios de 3.600 ha da Oldesa (Óleo de Dendê Ltda.), em Nazaré, BA, e em plantios de 1.700 ha pertencentes à Opalma (Óleo de Palma S.A.), em Taperoá, BA. De cada cacho, foi retirada uma amostra de vinte espiguetas, para análise da taxa de fecundação.

\section{Resultados e Discussão}

A média do percentual dos frutos normais, de cachos de dendezeiros da Esmai, foi $79,4 \pm 0,5 \%$; a percentagem mínima foi $20,1 \%$ e a máxima $96,8 \%$. A média dos frutos normais, por cacho, foi de $276 \pm 4,2$. Após introdução de E. kamerunicus no Sul da Bahia, a taxa de fecundação de março/2004 a março/2006 estava acima de 70\%, tendo-se mantido praticamente constante no período estudado (Figura 1). Considerando-se que a taxa média de fecundação, no presente trabalho, foi de 79,4\% e, admitindo-se como referência a taxa de fecundação de $60 \%$, antes da introdução de E. kamerunicus, verificouse o incremento de $19 \%$ na fecundação, após a introdução dessa espécie.

Estima-se que existam 20.000 ha de dendezeiros subespontâneos no Sul da Bahia (Mesquita, 2000). Esses dendezeiros, em sua maioria, são constituídos pela variedade Dura e, por se tratar de atividade extrativista, a produtividade é relativamente baixa $\left(4 \mathrm{Mg} \mathrm{ha}^{-1} \mathrm{de}\right.$ cachos por ano). Contudo, estima-se que o ganho em reais, após a introdução de E. kamerunicus no Sul da Bahia, tenha sido de aproximadamente $\mathrm{R} \$ 404.800,00$. Se a referida área fosse constituída de dendezeiros da variedade Tenera, e os plantios fossem conduzidos com tratos agronômicos adequados, os ganhos anuais com a introdução de E. kamerunicus poderiam ser da ordem de R \$1.500.000,00, somente com óleo de palmiste. Salienta-se, ainda, que frutos partenocárpicos têm muita fibra e pouco óleo no mesocarpo. Assim, com a taxa de fecundação elevada, têm-se também ganhos econômicos com o óleo de palma (mesocarpo) propriamente dito.

Nos plantios das empresas Oldesa e Opalma, nos municípios de Nazaré e Taperoá, registraram-se percentuais de frutos normais de 76,33 e 76,77\%, respectivamente (Tabela 1). Assim, considerando-se que já foi obtida em área da Oldesa a produtividade de $15 \mathrm{Mg}$ de cacho de dendê, isso corresponde a $300 \mathrm{~kg}$ de óleo de palmiste. Com base nos estudos de Maia (1977), que encontrou taxa de fecundação de $68 \%$ para os dendezeiros de Nazaré, verifica-se que houve acréscimo de $11 \%$ de frutos normais nos plantios da Oldesa, correspondente ao aumento de $33 \mathrm{~kg} \mathrm{ha}^{-1}$. Admitindo-se essa produtividade para a área total (3.600 ha) da Oldesa,

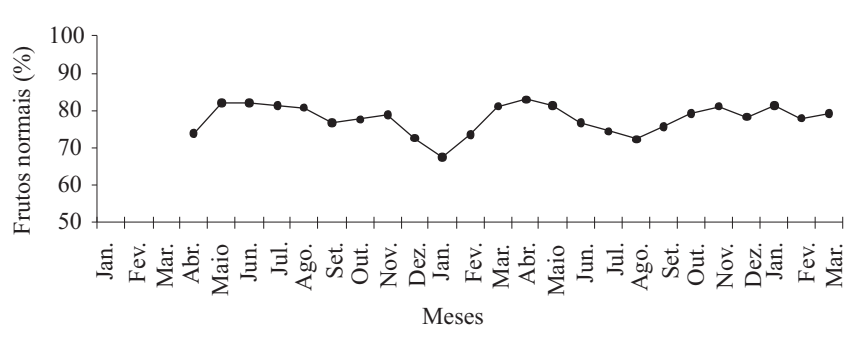

Figura 1. Taxa de fecundação de dendezeiros, após a introdução de Elaeidobius kamerunicus em Una, BA, no período de janeiro/2004 a março/2006. 
ter-se-ia ganho de aproximadamente 118,8 Mg de óleo de palmiste. Como o valor do óleo de palmiste, na região de Valença, BA, é de R \$2.300,00 a megagrama, estimase que o lucro anual da Oldesa deva ser de aproximadamente $\mathrm{R}$ \$273.240,00, após a introdução de E. kamerunicus na região.

Infelizmente, as empresas citadas não fazem análise física dos cachos de dendê com certa periodicidade em seus plantios. Já que a região de Nazaré e Taperoá estão circundadas por milhares de dendezeiros subespontâneos, é de se esperar que alguma variação ocorra na taxa de fecundação dos dendezeiros, principalmente ao se considerar que a distribuição de chuvas nessas regiões difere da de Una.

Nos últimos anos, o fruto de dendê 'Cafuné' praticamente desapareceu nos plantios subespontâneos, entre os municípios de Camamu e Nazaré, BA. Esses frutos ficam nas partes internas do cacho, que, quando retirado da planta, cai sobre o solo e os frutos internos desprendem-se com o impacto. O desaparecimento desses frutos está relacionado ao fato de os espécimes de E. kamerunicus penetrarem nas partes mais internas das inflorescências femininas em antese e as fecundarem.

Harun \& Noor (2002) estudaram a frutificação e componentes do cacho de dendê, na Malásia, e observaram que as mudanças nas características dos cachos, ocasionadas pela introdução de $E$. kamerunicus, estão relacionadas ao aumento da fertilização das flores internas. Nesse mesmo trabalho, a polinização assistida (manual) foi comparada com entomófila, e observou-se que, na primeira polinização, a taxa de fecundação variou de 2 a $90 \%$, enquanto na segunda, a taxa média de fecundação foi $80 \%$. Isto evidencia que a polinização efetuada por E. kamerunicus foi mais eficiente, pois, por penetrar nas partes internas do cacho, na busca por alimento, fecunda as flores (Harun \& Noor, 2002).

Tabela 1. Taxa média ( \pm erro-padrão) de fecundação de dendezeiros, das empresas Oldesa e Opalma.

\begin{tabular}{lccc}
\hline Empresa & $\begin{array}{c}\text { № de frutos } \\
\text { normais }\end{array}$ & $\begin{array}{c}\text { № de frutos } \\
\text { partenocárpicos }\end{array}$ & $\begin{array}{c}\text { Taxa de } \\
\text { fecundação }\end{array}$ \\
\hline Oldesa & $267,60 \pm 23,46$ & $82,95 \pm 22,31$ & $76,33 \pm 3,20$ \\
Opalma & $220,15 \pm 13,88$ & $66,60 \pm 10,06$ & $76,77 \pm 3,40$ \\
\hline
\end{tabular}

Na Malásia, Harun \& Noor (2002) trabalharam na faixa de $0-100 \%$ de frutos normais, e obtiveram ajuste quadrático, na relação peso e taxa de fecundação, significativo a $5 \%$ de probabilidade $\left(\mathrm{R}^{2}=0,4291\right)$. No presente trabalho, ao se utilizar o mesmo modelo polinomial, verificou-se que não houve relação entre peso médio dos cachos e taxa de fecundação (Figura 2). A explicação para esse resultado decorre de a faixa do percentual de frutos normais situar-se entre 60 e $100 \%$. Nessa mesma faixa, os resultados de Harun \& Noor (2002) também não apresentaram relação.

No período analisado, foram coletados 419.107 espécimes de E. kamerunicus e 114.021 espécimes de E. subvittatus, ou seja, a população de E. kamerunicus em Una quase quadruplicou em relação à de E. subvittatus (Figura 3).

Observou-se que E. kamerunicus foi mais numeroso que E. subvittatus nos municípios de Ituberá, Taperoá, Valença e Nazaré, exceto no Município de Itapebi (Tabela 2). No extremo Sul do Estado da Bahia, a freqüência de dendezeiros subespontâneos diminuiu consideravelmente. Assim, é possível que a ausência de hospedeiros, aliada à presença de extensas áreas com plantios de eucalipto, no entorno do Município de Itapebi, estejam contribuindo para a lenta colonização da região por E. kamerunicus.

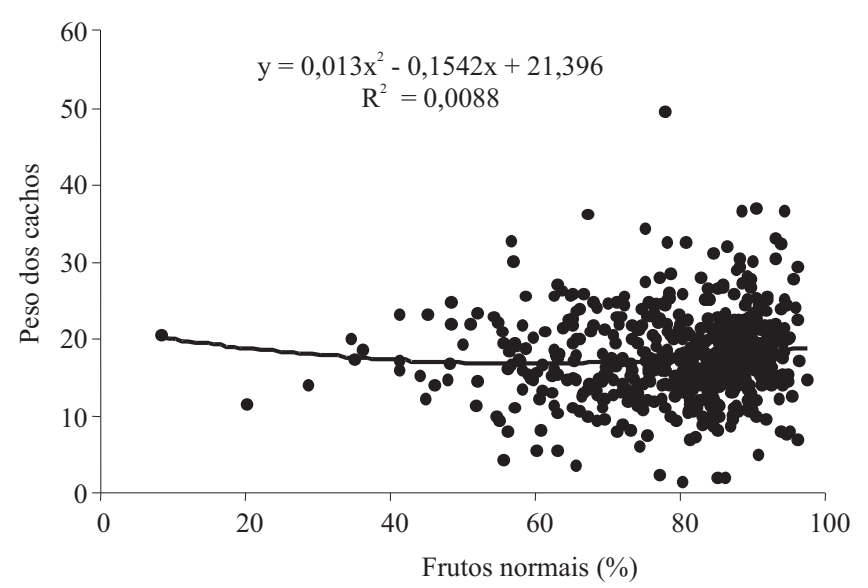

Figura 2. Ajuste polinomial da relação entre o percentual de frutos normais e o peso de cacho em dendezeiros. 
A distribuição de chuvas no Município de Una não afetou a atividade de vôo de E. subvittatus e E. kamerunicus sobre as inflorescências masculinas e femininas dos dendezeiros, o que fez com que a taxa de fecundação nos dendezeiros se mantivesse estável na Esmai (Figura 4).

Em plantios de dendezeiros na Colômbia, Genty et al. (1986) avaliaram a influência da precipitação na polinização entomófila e seu reflexo sobre a taxa de fecundação dos dendezeiros. Segundo os autores, nas regiões Norte e Oriental, as taxas de fecundação foram altas - entre 70 e 75\%. Na Região Ocidental, a taxa de fecundação foi de intermediária a baixa, próximo a $58 \%$, enquanto na Região Central foi crítica, em torno de $45 \%$. Essas diferenças parecem ter relação direta com populações de E. subvittatus. A Região Norte, por ter baixa precipitação anual $(1.400 \mathrm{~mm})$, apresentou sempre populações elevadas de E. subvittatus. A Região Oriental, apesar das precipitações altas $(3.500 \mathrm{~mm})$, apresentou período de estiagem longo, o que favoreceu o desenvolvimento de E. subvittatus. Na Região Ocidental (Costa do Pacífico), por haver altíssimas

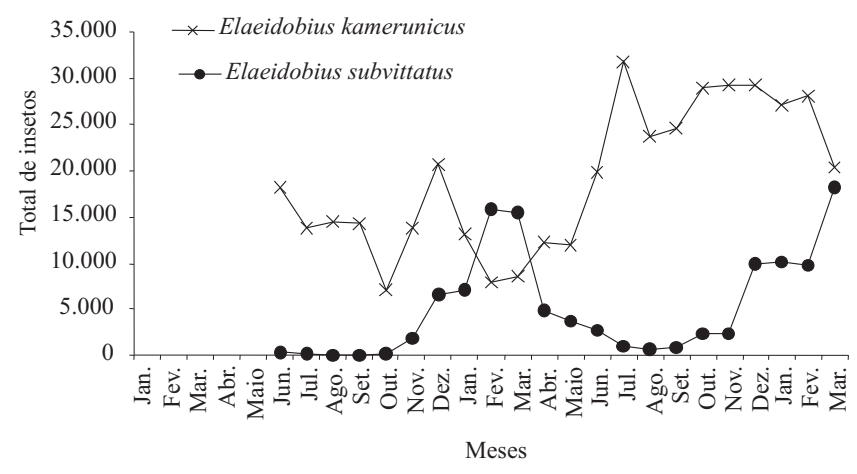

Figura 3. Flutuação mensal de Elaeidobius kamerunicus e Elaeidobius subvittatus, sobre a inflorescência masculina do dendezeiro em antese, no período de janeiro/2004 a março/2006.

Tabela 2. Total e percentual de Elaeidobius kamerunicus e Elaeidobius subvittatus, coletados em setembro/2006 em plantios de dendezeiros, nos municípios de Ituberá, Taperoá, Valença, Nazaré e Itapebi, BA.

\begin{tabular}{lccccc}
\hline Espécie & Ituberá & Taperoá & Valença & Nazaré & Itapebi \\
\hline E. kamerunicus & $2.555(82 \%)$ & $3.214(81 \%)$ & $3.578(90 \%)$ & $2.020(72 \%)$ & $763(32 \%)$ \\
\hline E. subvittatus & $554(18 \%)$ & $734(19 \%)$ & $66(10 \%)$ & $770(28)$ & $1.615(68 \%)$ \\
\hline
\end{tabular}

precipitações $(4.100 \mathrm{~mm})$, a presença de E. subvittatus foi reduzida, tendo sido compensada pela a atividade polinizadora de M. costaricensis.

Mariau \& Genty (1988) citam que, no Estado do Pará, no período chuvoso, foi observada uma diminuição expressiva da atividade polinizadora de E. subvittatus. Segundo esses autores, tal fato pode estar relacionado a um fungo sobre as inflorescências masculinas, depois do estádio de antese, razão pela qual a taxa de fecundação pode ter ficado abaixo de $30 \%$ em média, como ocorrido em outubro de 1983. De acordo com os autores, a atividade polinizadora do E. subvittatus foi bem maior na estação seca - período em que os insetos polinizam as flores -, e a taxa de fecundação chegou a $80 \%$.

Em Una, observou-se uma relação mais forte de E. kamerunicus com a distribuição de chuva mensal (Figura 5). O número de espécimes de E. kamerunicus observados entre agosto/2004 e fevereiro/2005, período com variações extremas de chuva (média de 93,7 mm), contrastou com o do período de junho/2005 a março/2006, quando a precipitação mensal atingiu $160,8 \mathrm{~mm}$. O total de insetos quase triplicou, tendo passado de 91.343 espécimes de agosto/2004 a fevereiro/2005 para 262.855 espécimes em junho/2005 a março/2006.

A densidade populacional de E. subvittatus pode ser influenciada pela variação da temperatura do ambiente (Figura 5). E. subvittatus apresentou comportamento mais ativo nos meses quentes $(r=0,82 ; p<0,05)$ e, nos

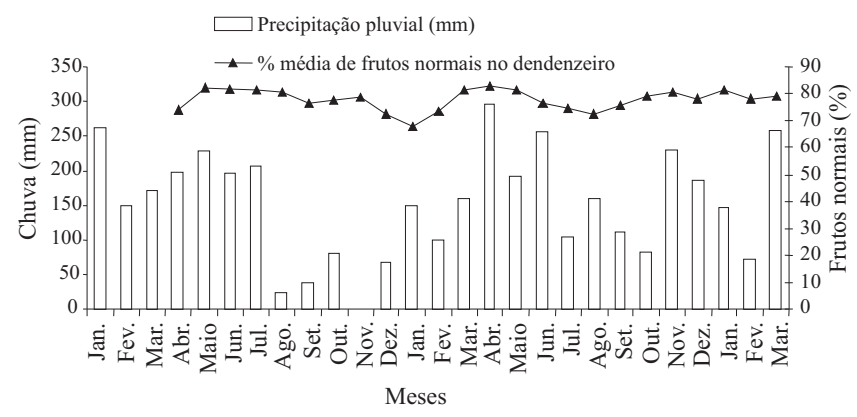

Figura 4. Precipitação pluvial e percentual de frutos normais, no Município de Una, BA, no período de janeiro/2004 a março/2006. 


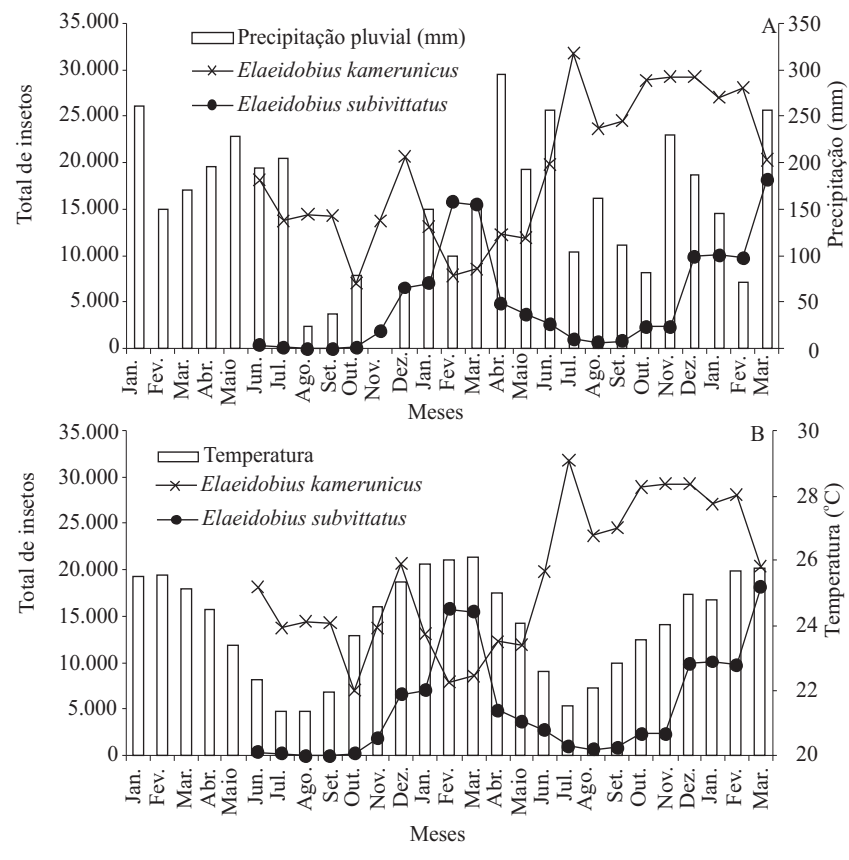

Figura 5. Precipitação pluvial e total de insetos Elaeidobius kamerunicus e Elaeidobius subvittatus (A) e temperatura média mensal $\left({ }^{\circ} \mathrm{C}\right)$ e total de insetos E. kamerunicus e E. subvittatus (B), no Município de Una, BA, no período de janeiro/2004 a março/2006.

meses de inverno sua população declinou, nos dois anos avaliados. A espécie E. kamerunicus não foi tão afetada pela variação da temperatura do ambiente $(\mathrm{r}=-0,15$; $\mathrm{p}<0,05)$. Assim, E. subvittatus deve competir com E. kamerunicus, nos meses mais quentes do ano, por espaço para oviposição e alimentação de pólen.

\section{Conclusões}

1. A introdução de Elaeidobius kamerunicus contribui para incrementar a taxa de fecundação dos dendezeiros.

2. Elaeidobius kamerunicus é espécie dominante, no Sul da Bahia, em relação a Elaeidobius subvittatus.

3. Elaeidobius subvittatus tem comportamento menos ativo no inverno.

\section{Agradecimentos}

À Embrapa Amazônia Oriental, pelo apoio na introdução de Elaeidobius kamerunicus em Una, BA; à Ceplac, pelo apoio financeiro; a Fernando Santana de Miranda, pela análise física dos cachos; a Solange Pereira Gavazza Rufino, pela digitação dos dados; a Edmundo Souza Lima, pela contagem dos insetos; a Sinval de Souza Pinto, pelo suporte de infraestrutura; ao Dr. José Roberto Vieira e ao Dr. Jonas de Souza, pelas críticas e sugestões.

\section{Referências}

CHINCHILLA, C.; ESCALANTE, M.; RICHARDSON, D.L. Polinización en palma aceitera (Elaeis guineensis Jacq.) en Centroamérica: II. Comportamento de insetos. Turrialba, v.40, p.461-470, 1990.

CONCEIÇÃO, H.E.O.; MULLER, A.A. Botânica e morfologia do dendezeiro. In: VIÉGAS, I.J.M.; MULLER, A.A. (Ed.). A Cultura do dendezeiro na Amazônia brasileira. Belém: Embrapa Amazônia Oriental, 2000. p.31-44.

GENTY, P.; GARZON, A.; LUCCHINI, F.; DELVARE, C. Polinización entomófila de la palma africana en América tropical. Oléagineux, v.41, p.99-112, 1986.

HARUN, M.H.; NOOR, M.R. Fruit set and oil palm bunch components. Journal of Oil Palm Research, v.14, p.24-33, 2002.

MAIA, A.S. Características do dendezeiro subespontâneo na Bahia. Cruz das Almas: Ipeal, 1977. 8p. (Ipeal. Comunicado técnico, 57).

MARIAU, D.; GENTY, P.H. Contribución de IRHO al studio de los insectos polinizadores de la palma de aceite en África, América del Sur e Indonesia. Palmas, v.9, p.33-38, 1988.

MESQUITA, A.S. Do azeite de dendê de Ogum ao palm oil commodity: uma oportunidade que a Bahia não pode negar. Bahia Agrícola, v.5, p.22-27, 2002.

PRADA, M.; MOLINA, D.; VILLARREL, D.; BARRIOS, R.A. Efectividad de dos especies del género Elaeidobius (Coleoptera: Curculionidae) como polinizador en palma aceitera. Bioagro, v.10, p.3-10, 1998.

SYED, R.A.; LAW, I.H.; CORLEY, R.H.V. Insect pollination of oil palm: introduction, establishment and pollinating efficiency of Elaeidobius kamerunicus in Malaysia. Planter, v.58, p.547-561, 1982. 